Proceedings of the 2010 Winter Simulation Conference

B. Johansson, S. Jain, J. Montoya-Torres, J. Hugan, and E. Yücesan, eds.

\title{
CAPTURING PARAMETER UNCERTAINTY IN SIMULATIONS WITH CORRELATED INPUTS
}

Bahar Biller

Tepper School of Business

Carnegie Mellon University

Pittsburgh, PA 15213, U. S. A
Canan Gunes

Tepper School of Business

Carnegie Mellon University

Pittsburgh, PA 15213, U. S. A

\begin{abstract}
We consider a stochastic simulation with correlated inputs represented by a multivariate normal distribution. The objectives are to (i) account for parameter uncertainty (i.e., the uncertainty around the multivariate normal distribution parameters estimated from finite historical input data) in the mean performance estimate and the confidence interval of the simulation; and (ii) decompose the total variation of the simulation output into distinct terms representing stochastic and parameter uncertainties. We describe how to achieve these objectives using the Bayesian model of Biller and Gunes (2010) for capturing parameter uncertainty and the Bayesian simulation replication algorithm of Zouaoui and Wilson (2003) for output variance decomposition. We conclude with the extension of this study to arbitrary marginal distributions and dependence measures with positive tail dependencies.
\end{abstract}

\section{INTRODUCTION}

The common practice in the design of stochastic simulations is to estimate the input distribution parameters from historical data sets of finite length and drive the simulations with the random variates generated from the estimated input distributions. This practice, however, ignores the uncertainty around the estimated parameters (i.e., parameter uncertainty (Raftery et al. 1996)), and accounts only for stochastic uncertainty (i.e., the uncertainty that arises from the dependence of the output on the simulation's random input streams (Helton 1997)) in the output analysis. Consequently, the simulation analyst often obtains inaccurate estimates for mean performance measures and low coverage for confidence intervals. For example, Hayes (1969) discusses the shortcomings of ignoring demand parameter uncertainty in inventory planning; i.e., plugging the maximum likelihood estimates of the demand parameters obtained from limited historical demand data into the formulas of the order-up-to levels. Chick (2001) shows that accounting for parameter uncertainty in the simulation of an M/M/1 queueing system improves the estimate of the average number in queue and the average percent availability of the server. In another study, Zouaoui and Wilson (2004) demonstrate that the parameter uncertainty accounts for $80 \%$ of the total uncertainty around the mean waiting time estimate of an M/G/1 queuing system; therefore, ignoring parameter uncertainty leads to erroneous point estimates and confidence intervals of the mean waiting time. In a more recent paper, Biller and Gunes (2010) illustrate the importance of accounting for parameter uncertainty in the simulation of a multi-product inventory system with correlated demands.

There exists a well-established literature about representing parameter uncertainty in discrete-event stochastic simulation outputs; see Cheng and Holland (1997), Barton and Schruben (2001), Chick (1999, 2001), Zouaoui and Wilson (2003, 2004), Ng and Chick (2006), and Biller and Gunes (2010) for example studies. Specifically, Chick $(1999,2001)$ is the first to use the Bayesian Model Averaging (BMA) approach (Cooke 1994) for capturing stochastic and parameter uncertainties. Chick's formulation leads to the simulation repli- 
cation algorithm that allows the representation of parameter uncertainty by sampling input distributions and their parameters from Bayesian posterior density functions before each simulation replication. The simulation replication algorithm has been used recently by Biller and Gunes (2010) to account for stochastic uncertainty and parameter uncertainty in the confidence intervals of simulations with correlated inputs. Specifically, Biller and Gunes (2010) let the correlated inputs be represented by the flexible Normal-To-Anything (NORTA) distribution (Cario and Nelson 1997) and use Sklar's marginal-copula representation (Nelsen 1999) to develop a Bayesian model for sampling NORTA parameters (i.e., the marginal-distribution parameters and the correlation matrix) from their Bayesian posterior density functions. Biller and Gunes (2010) further use Cooke's copula-vine specification (Kurowicka and Cooke 2006) to construct the correlation matrix using a mix of unconditional and conditional pairwise correlations instead of using only unconditional pairwise correlations. This eliminates the need to use constraints for insuring the positive definiteness of the correlation matrix to be sampled in a system with more than two correlated inputs. In this paper, we represent the correlated inputs of our simulation with the multivariate normal distribution, which is a special case of the NORTA distribution obtained by setting each marginal distribution of NORTA to the normal distribution. First, we use the Bayesian model of Biller and Gunes (2010) to account for parameter uncertainty in the simulation outputs. Then, we decompose the simulation output variance into variances due to stochastic and parameter uncertainties. Since the simulation replication algorithm used by Chick (2001) and Biller and Gunes (2010) does not allow us to obtain such a decomposition of the simulation output variance, we use the Bayesian simulation replication algorithm (BSRA) introduced by Zouaoui and Wilson (2003) for this purpose.

The rest of the paper is organized as follows. In Section 2, we describe how to use the Bayesian model developed by Biller and Gunes (2010) for representing parameter uncertainty in a stochastic simulation with normally distributed, correlated inputs. In Section 3, we incorporate this Bayesian model into the Bayesian simulation replication algorithm of Zouaoui and Wilson (2003). In Section 4, we describe how to decompose the variance of the simulation output into terms related to stochastic uncertainty and parameter uncertainty. We discuss the extension of this study to arbitrary marginal distributions in Section 5.1 and dependence structures with positive tail dependencies in Section 5.2. We conclude with a summary of results in Section 6.

\section{A BAYESIAN MODEL FOR REPRESENTING PARAMETER UNCERTAINTY}

We consider a stochastic simulation with two correlated inputs in Section 2.1, and a simulation with three correlated inputs in Section 2.2. We extend the number of simulation inputs to $k>3$ in Section 2.3. In each section, we assume that the $k$ correlated inputs $X_{i}, i=1,2, \ldots, k$ have a $k$-dimensional normal distribution with parameters $i, i=1,2, \ldots, k ; \quad{ }_{i}^{2}, i=1,2, \ldots, k$; and $k=[(i, j) ; i, j=1,2, \ldots, k]$. Specifically, $i$ and

${ }_{i}^{2}$ denote the mean and the variance of the $i^{\text {th }}$ simulation input, while ${ }_{k}$ is the $k$-dimensional correlation matrix with $(i, j)$ denoting the product-moment correlation between random variables $X_{i}$ and $X_{j}$.

\subsection{Simulations with Two Correlated Inputs}

The objective of this section is to sample the parameter $i$ and the parameter $i_{i}$ of the normally distributed random variable $X_{i}$ for $i=1,2$ as well as the product-moment correlation between $X_{1}$ and $X_{2}$. Following Biller and Gunes (2010) and using Sklar's theorem (Sklar 1959), we write the likelihood function $L(\mathbf{x} \mid 1,1,2, \quad 2, \quad$ ) for the two-dimensional historical input data $\mathbf{x}=\left\{x_{i, t} ; i=1,2, t=1,2, \ldots, n\right\}$ of length $n$ as a multiplication of the marginal normal density functions and a two-dimensional copula density function, where represents the probability density function of a standard normal random variable:

$$
\begin{aligned}
L(\mathbf{x} \mid 1, \quad 1,2,2, \quad) & ={ }_{i=1 t=1}^{2} \frac{1}{i}\left(\frac{x_{i, t}-i}{i}\right) \\
& \times{ }_{t=1}^{n} \frac{1}{\sqrt{1-2}} \exp \left\{\frac{-2\left(\frac{x_{1, t}-1}{1}\right)^{2}-2\left(\frac{x_{2, t}-2}{2}\right)^{2}+2\left(\frac{x_{1, t}-1}{1}\right)\left(\frac{x_{2, t}-2}{2}\right)}{2(1-2)}\right\}
\end{aligned}
$$

Next, we choose prior density functions for the distribution parameters $1,1,2,2$, and . Following Biller and Gunes (2010), we use the conjugate inverted Wishart prior density function with zero degrees of 
freedom for the correlation matrix $2=\left[\begin{array}{lll}1 & ; & 1\end{array}\right]$; i.e., $\quad(2) \propto\left|{ }_{2}\right|^{-3 / 2}$. This is a diffuse prior density function coinciding with the beta prior density function of Barnard et al. (2000). We also choose conjugate prior density functions $(1,1)$ and $(2,2)$ for the marginal-distribution parameters $i$ and $1 /{ }_{i}^{2} \equiv_{i}$ for $i=1,2$; they are the normal-gamma density functions given by

$$
(i, i)=\frac{\left(s_{0 i} i\right)^{1 / 2}}{\sqrt{2}} \exp \left\{-\left({ }_{i}-0 i\right)^{2} s_{0 i} i / 2\right\} \frac{0 i{ }^{0 i}}{(0 i)} i^{0 i-1} \exp \{-0 i i\},
$$

where $0_{i}, s_{0 i}, \quad 0 i$, and $0_{0 i}$ are the prior distribution parameters for $i=1,2$ (Bernardo and Smith 1994).

The multiplication of the prior density functions $(1,1),(2,2)$, and $(2)$ with the likelihood function $L(\mathbf{x} \mid 1,1,2, \quad 2, \quad)$ leads to the following joint posterior density function

$$
\begin{aligned}
& \overbrace{\frac{\left(s_{n 1} 1\right)^{1 / 2}}{\sqrt{2}} \exp \left\{-(1-n 1)^{2} s_{n 1}{ }_{1} / 2\right\} \frac{n 1}{\left({ }_{n 1}\right)} 1^{n 1-1} \exp \left\{\begin{array}{lll}
- & & \\
\times &
\end{array}\right\}}^{h\left({ }_{1},{ }_{1} \mid \mathbf{x}\right)}
\end{aligned}
$$

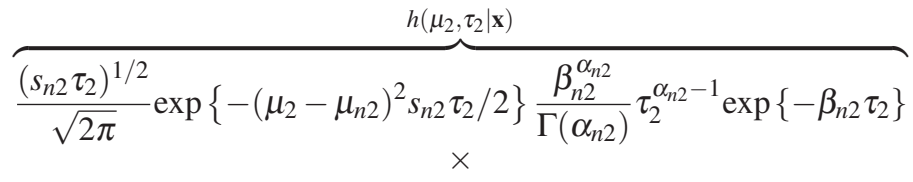

$$
\begin{aligned}
& \overbrace{|2|^{-(3+n) / 2} \operatorname{etr}\left(-\frac{1}{2}\left[\mathbf{S}{ }_{2}^{-1}-\mathbf{S}\right]\right)}^{h\left({ }_{2} \mid{ }_{1}, 2,{ }_{1},{ }_{2}, \mathbf{x}\right)},
\end{aligned}
$$

where

$$
\mathbf{S}={ }_{t=1}^{n}\left(\begin{array}{c}
\frac{x_{1, t}-1}{x_{2, t}-1} \\
2
\end{array}\right)\left(\begin{array}{c}
\frac{x_{1, t}-1}{x_{2, t}-1} \\
2
\end{array}\right)^{\prime},
$$

etr is the exponent of the trace operator, ${ }_{n i}=\left(\begin{array}{ll}s_{0 i} & 0 i\end{array}\right) /\left(s_{0 i}+n\right)+\left(x_{i} /\left(s_{0 i}+n\right)\right), s_{n i}=s_{0 i}+n, \quad{ }_{n i}={ }_{0 i}+n / 2$, and ${ }_{n i}=0 i+{ }_{i=1}^{n}\left(x_{i}-x_{i} / n\right)^{2} / 2+s_{0 i} n\left(0_{0 i}-x_{i} / n\right) /\left(s_{0 i}+n\right)$ for $i=1,2$. What is important to recognize in (2) is that Sklar's marginal-copula representation allows us to write the joint posterior density function as a product of the marginal posterior density functions and the posterior copula density function, which are proportional to $h\left({ }_{1}, 1 \mid \mathbf{x}\right), h\left({ }_{2}, 2 \mid \mathbf{x}\right)$, and $h\left({ }_{2} \mid 1_{1}, 2,1,2, \mathbf{x}\right)$ of (2), respectively. Therefore, we first sample $i$ and ${ }_{i}$ from the normal-gamma posterior density function $h\left(i,{ }_{i} \mid \mathbf{x}\right)$ independently for $i=1,2$. Then, we sample the correlation matrix 2 from the inverted Wishart posterior copula density function $h\left({ }_{2} \mid 1,2,1,2, \mathbf{x}\right)$. The generation of the parameters $i$ and $i$ from the normal-gamma posterior density function $h\left({ }_{i},{ }_{i} \mid \mathbf{x}\right)$ is easily accomplished by first generating the parameter ${ }_{i}$ from a gamma distribution with parameters $\quad n i$ and ${ }_{n i}$ and then generating the parameter ${ }_{i}$ from a normal distribution with mean $n i$ and variance $1 /\left(s_{n i} i\right)$. The sampling of the correlation matrix from the inverted Wishart posterior copula density function $h\left(\begin{array}{l}2 \\ 2\end{array} 1,2,1,2, \mathbf{x}\right)$ with parameters $n$ and $\mathbf{S}$ starts with the generation of random variates $a, b$, and $c$ from a chi-square distribution with $n$ degrees of freedom, a standard normal density function, and a chi-square distribution with $n-1$ degrees of freedom, respectively. We then construct a two-dimensional matrix $\mathbf{T}_{2}=\left[\begin{array}{lll}\sqrt{a} & 0 ; b \sqrt{c}\end{array}\right]$, determine the matrix $\mathbf{W}_{2}$ that satisfies $\mathbf{W}_{2} \mathbf{W}_{2}^{\prime}=\mathbf{S}^{-1}$, and construct $\mathbf{C}_{2}=\mathbf{T}_{2}^{\prime} \mathbf{W}_{2}$. We obtain a value for 2 as a result of computing $\mathbf{C}_{2}^{-1}\left(\mathbf{C}_{2}^{-1}\right)^{\prime}$ (Section A.3 of Biller and Gunes (2010)).

\subsection{Simulations with Three Correlated Inputs}

As a result of extending the discussion in Section 2.1 to a three-dimensional setting, we obtain a model that independently samples marginal-distribution parameters $i$ and $i$ from the normal-gamma posterior density function $h(i, i \mid \mathbf{x})$ for $i=1,2,3$. Then, we sample the three-dimensional correlation matrix $3=$ $\left[\begin{array}{lll}1 & (1,2) \quad(1,3) ;(2,1) 1 \quad(2,3) ; \quad(3,1)\end{array}\right.$ 
$(3,2) 1]$ from its posterior density function denoted by $h(3 \mid 1,2,3,1,2,3, \mathbf{x})$. However, the derivation of a joint posterior density function for 3 is complicated by the need to insure its positive definiteness. To overcome this particular challenge associated with the positive definiteness of ${ }_{3}$, Biller and Gunes (2010) apply the copula-vine specification of Kurowicka and Cooke (2006) to the three-dimensional copula density function. Specifically, a vine is a graphical model that is used to construct multivariate distributions using two dimensional conditional and unconditional distributions (Kurowicka and Cooke 2006). Following Biller and Gunes (2010), we use a $C$-vine for representing the normal copula density function of the random variables $X_{1}, X_{2}$, and $X_{3}$; we refer the reader to Kurowicka and Cooke (2006) for an illustration of different vines.

The application of the $C$-vine specification allows us to represent the dependence structure of the underlying input process, which has been characterized by correlations $(1,2),(1,3)$, and $(2,3)$ in $h(3 \mid 1,2,3,1,2,3, \mathbf{x})$, in terms of (unconditional) correlations $(1,2)$ and $(1,3)$, and conditional correlation $(2,3 ; 1)$. More specifically, $(2,3 ; 1)$ is the correlation between $X_{2}$ and $X_{3}$ conditional on $X_{1}$; i.e., $X_{2} \mid X_{1}$ and $X_{3} \mid X_{1}$. Since we represent the joint distribution of the random variables $X_{1}, X_{2}$, and $X_{3}$ by a normal distribution, the conditional correlation $(2,3 ; 1)$ is also the partial correlation defined as the correlation between the orthogonal projections of $X_{2}$ and $X_{3}$ on the plane orthogonal to the space spanned by $X_{1}$. Therefore, the partial correlation $(2,3 ; 1)$ is given by $((1,2)-(1,3)(2,3)) / \sqrt{\left(1-{ }^{2}(1,3)\right)(1-2(2,3))}$ (Kurowicka and Cooke 2006).

The joint use of Sklar's marginal-copula representation and Cooke's copula-vine specification for the characterization of our three-dimensional input process suggests that priors for the marginal-distribution parameters $i$ and $i, i=1,2,3$, and the two-dimensional correlation matrices $2(1,2)=\left[\begin{array}{ll}1 & (1,2) ;(1,2) 1\end{array}\right]$,

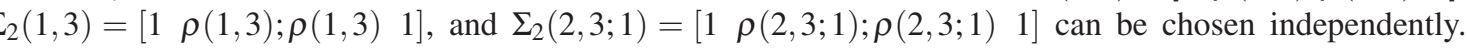
Specifically, the prior density functions $(i, i), i=1,2,3$ are normal-gamma conjugate priors. Assuming the probabilistic independence of the correlation matrices ${ }_{2}(1,2),{ }_{2}(1,3)$, and ${ }_{2}(2,3 ; 1)$, we choose their prior density functions as proportional to the inverted Wishart density functions; i.e, $\quad(2(1,2)) \propto|2(1,2)|^{-3 / 2}$, $(2(1,3)) \propto\left|{ }_{2}(1,3)\right|^{-3 / 2}$, and $(2(2,3 ; 1)) \propto\left|{ }_{2}(2,3 ; 1)\right|^{-3 / 2}$. The multiplication of these prior density functions with the likelihood function of the available input data results in a joint posterior density function which can be written as a multiplication of the marginal normal-gamma posterior density functions $h(i, i \mid \mathbf{x}), i=1,2,3$, and the conditional and unconditional inverted Wishart posterior copula density functions $h(2(1,2) \mid 1,2, \quad 1,2, \mathbf{x}), h(2(1,3) \mid 1,3,1,3, \mathbf{x})$, and $h(2(2,3 ; 1) \mid i, i, i=1,2,3,2(1,2), 2(1,3), \mathbf{x})$. Consequently, we sample the marginal-distribution parameters $i$ and $i$ from $h(i, i \mid \mathbf{x}), i=1,2,3$ as discussed in Section 2.1, and the two-dimensional correlation matrices ${ }_{2}(1,2), \quad 2(1,3)$, and $2(2,3 ; 1)$ from $h(2(1,2) \mid 1,2,1,2, \mathbf{x}), h(2(1,3) \mid 1,3,1,3, \mathbf{x})$, and $h\left(2(2,3 ; 1) \mid i, i, i=1,2,3,2(1,2),{ }_{2}(1,3), \mathbf{x}\right)$, respectively. More specifically, we first sample $2_{2}(1,2)$ and ${ }_{2}(1,3)$ independently from their inverted Wishart posterior density functions with parameters $n$ and $\mathbf{S}(1, i), \mathrm{i}=2,3$ where

$$
\mathbf{S}(1, i)={ }_{t=1}^{n}\left(\frac{\frac{x_{1, t}-1}{x_{i, t}-1}}{i}\right)\left(\begin{array}{c}
\frac{x_{1, t}-1}{x_{i, t}-1} \\
i
\end{array}\right)^{\prime} .
$$

Then, we use the sampled values of $(1,2)$ and $(1,3)$ (i.e., $\sim(1,2)$ and $\sim(1,3))$ to sample $2(2,3 ; 1)$. More specifically, we sample $2(2,3 ; 1)$ from an inverted Wishart density function with parameters $n$ and $\mathbf{S}(2,3 ; 1)$, where

$$
\mathbf{S}(2,3 ; 1)={ }_{t=1}^{n}\left(\begin{array}{l}
\frac{\frac{x_{2, t^{-}}-2}{2}-2 \mid 1}{2 \mid 1} \\
\frac{\frac{x_{3, t^{-}} 3}{3}-3 \mid 1}{3 \mid 1}
\end{array}\right)\left(\begin{array}{l}
\frac{\frac{x_{2, t^{-}}-t_{2}}{2}-2 \mid 1}{2 \mid 1} \\
\frac{x_{3, t^{3}} 3}{3}-3 \mid 1 \\
3 \mid 1
\end{array}\right)^{\prime}
$$

and $\quad i \mid 1=\sim(1, i)\left(x_{1, t}-1\right) / \quad 1, \quad \stackrel{2}{i \mid 1}=1-{ }^{2}(1, i)$ for $i=2,3$. 


\subsection{Simulations with $k>3$ Correlated Inputs}

In the case of a stochastic simulation with $k>3$ correlated inputs, we select a normal-gamma prior density function for the marginal-distribution parameters of each component, extend the copula-vine specification used for the three-dimensional input process to the $k$-dimensional input process as described in Kurowicka and Cooke (2006), and assume inverted Wishart priors for all two-dimensional unconditional and conditional correlation matrices associated with the $C$-vine. Specifically, the $C$-vine for the $k$-dimensional input process is composed of $(k-1)$ different trees. The first tree contains correlations $(1, i), i=2,3, \ldots, k$, while the second tree is composed of partial correlations $(2, i ; 1), i=3,4, \ldots, k$. Finally, the $(k-1)^{\text {th }}$ tree includes the partial correlation $(k-1, k ; 1,2, \ldots, k-2)$. This allows us to write the joint posterior density function as the product of the $k$ normal-gamma marginal posterior density functions and $k(k-1) / 2$ two-dimensional unconditional and conditional inverted Wishart posterior copula density functions. Therefore, we first sample the marginal-distribution parameters of each component from its normal-gamma posterior density functions as described in Section 2.1. Then, we sample each of the (unconditional and conditional) two-dimensional correlation matrices from the inverted Wishart posterior density functions as described in Section 2.2. A detailed sampling algorithm for a five-dimensional NORTA distribution can be found in Biller and Gunes (2010).

\section{BAYESIAN SIMULATION REPLICATION ALGORITHM}

Zouaoui and Wilson (2003) use the Bayesian simulation replication algorithm (BSRA) for representing the stochastic uncertainty and the parameter uncertainty in the confidence intervals of the stochastic simulations with independent inputs. The structure of the Bayesian model in Section 2 allows us to use the BSRA in a similar manner to represent the stochastic and parameter uncertainties in the confidence intervals of stochastic simulations with correlated inputs. Letting $Y$ be a figure of merit whose mean is relevant to the decision making process, we incorporate the Bayesian model of Section 2 into the Bayesian simulation replication algorithm with the purpose of obtaining a point estimate and a confidence interval of the mean posterior response $E_{Y \mid \mathbf{x}}(Y \mid \mathbf{x})$.

We provide the Bayesian simulation replication algorithm for our $k$-dimensional input process in Figure 1. We use $i, i=1,2, \ldots, k$ to represent the vectors of the marginal distribution parameters; i.e., $i \equiv(\quad i, i)$, and ${ }_{i}^{m_{i}}$ to denote the marginal-distribution parameters sampled in the $m_{i}^{\text {th }}$ replication. Similarly, represents all (conditional and unconditional) two-dimensional correlation matrices of the $C$-vine specification; i.e., is composed of $2(1, i), i=2,3, \ldots, k$, and $2(j-1, i ; 1,2, \ldots, j-2), i=j, j+1, \ldots, k, j=3,4, \ldots, k . \quad d$, on the other hand, denotes all (conditional and unconditional) correlation matrices sampled in the $d^{\text {th }}$ replication. Furthermore, we use notation $\mathbf{x}=\left\{x_{i, t} ; i=1,2, \ldots, k, t=1,2, \ldots, n\right\}$ for the $k$-dimensional historical input data of length $n$, and $y_{m_{1}, m_{2}, \ldots, m_{k}, d, r}$ for the output response from the $r^{\text {th }}$ simulation run using the random-number input $\mathbf{u}^{r}$ and the sampled input parameters ${ }_{i}^{m_{i}}, i=1,2, \ldots, k$, and $d$.

The first (i.e., the most outer) loop estimates the uncertainty around the parameters 1 and 1 of the marginal distribution of the first component, while the second loop estimates the uncertainty around the parameters 2 and 2 of the marginal distribution of the second component. Similarly, the $k^{\text {th }}$ loop estimates the uncertainty around the parameters $k$ and $k$ of the marginal distribution of the $k^{\text {th }}$ component. The $(k+1)^{\text {th }}$ loop, on the other hand, estimates the uncertainty around the dependence parameters, while the $(k+2)^{\text {th }}$ (i.e., the most inner) loop estimates the stochastic uncertainty. Due to the use of the copula-vine specification to represent the uncertainty around the dependence parameter , it is possible to decompose this uncertainty into parts associated with individual correlations and partial correlations. However, for ease of presentation, we represent the uncertainty around in a single loop in Figure 1.

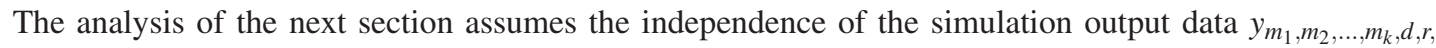
$m_{i}=1,2, \ldots, M_{i}, i=1,2, \ldots, k, d=1,2, \ldots, D$, and $r=1,2, \ldots, R$; therefore, we need to check whether output data satisfies this assumption. If we conclude dependency, then a number of methods including the batching method can be used to analyze the dependent output data (Law 2007). We refer the reader to Biller and Gunes (2010) for a detailed discussion on the independent sampling of the NORTA parameters and the analysis of the possibly dependent simulation output data for the proper execution of the simulation replication algorithm. 


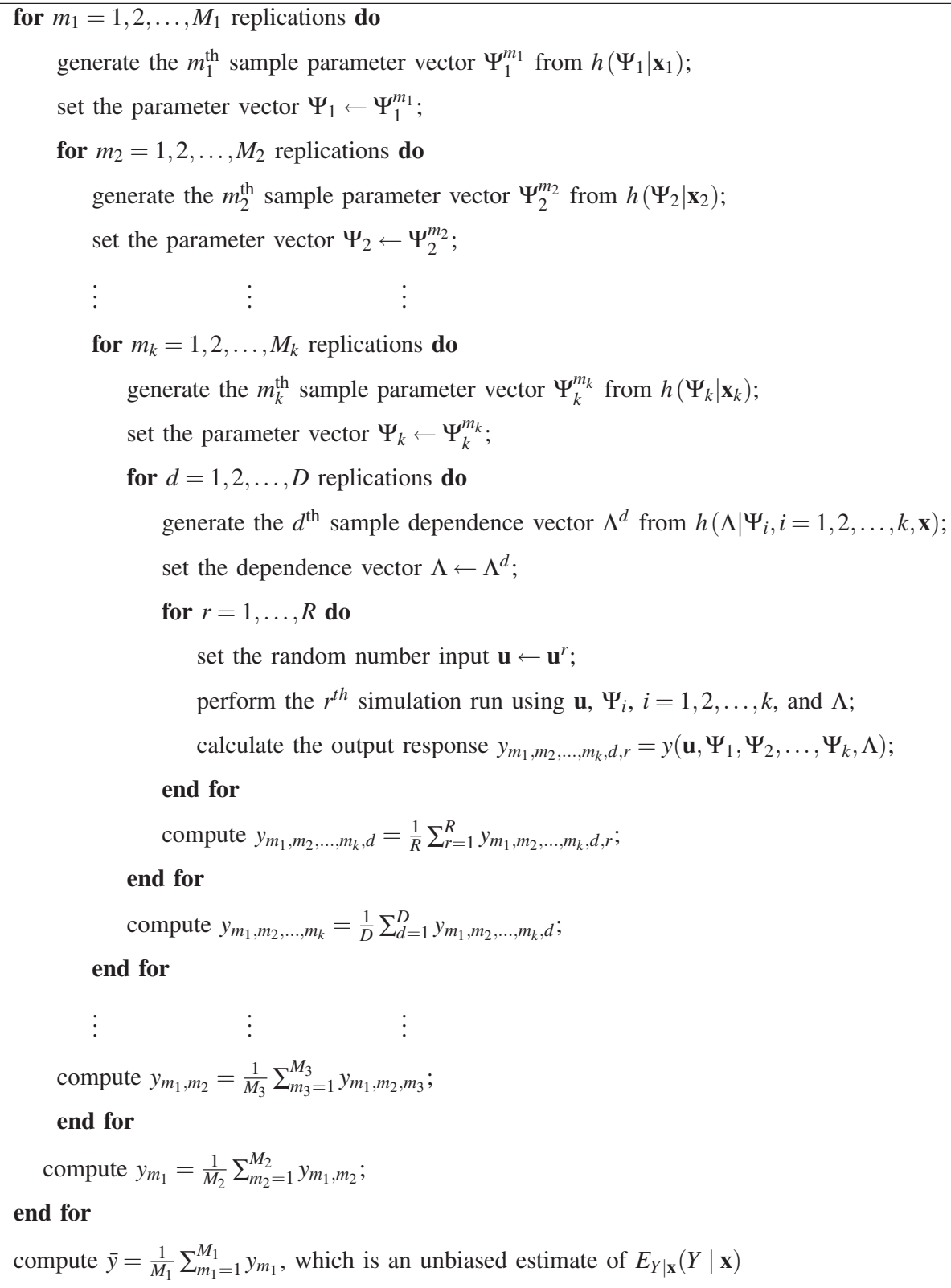

Figure 1: Bayesian simulation replication algorithm for the $k$-dimensional input process.

\section{OUTPUT VARIANCE DECOMPOSITION}

In this section, we use the output data obtained from the execution of the BSRA, and estimate both a point estimate for the mean posterior response $E_{Y \mid \mathbf{x}}(Y \mid \mathbf{x})$ and a posterior response variance as a function of stochastic uncertainty $\left({ }^{2}\right)$, dependence-parameter uncertainty $\left({ }^{2}\right.$ ), and marginal-distribution uncertainty for each simulation input $\left({ }^{2}, i=1,2, \ldots, k\right)$. Following Zouaoui and Wilson (2003), we express the output response from the $r^{\text {th }}$ simulation run as follows:

$$
\begin{aligned}
& y_{m_{1}, m_{2}, \ldots, m_{k}, d, r}=y\left(\mathbf{u}^{r}, \quad \begin{array}{l}
m_{1} \\
1
\end{array}, \begin{array}{l}
m_{2} \\
2
\end{array}, \ldots, \quad \begin{array}{l}
m_{k} \\
k
\end{array}, d\right)
\end{aligned}
$$

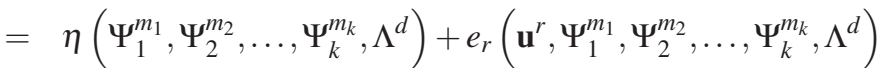




\section{Biller and Gunes}

The error $e_{r}\left(\mathbf{u}^{r}, \quad{ }_{1}^{m_{1}},{ }_{2}^{m_{2}}, \ldots, \quad{ }_{k}^{m_{k}},{ }^{d}\right)$ is the deviation of the simulation output $y_{m_{1}, m_{2}, \ldots, m_{k}, d, r}$ from the responsesurface $\left({ }_{1}^{m_{1}},{ }_{2}^{m_{2}}, \ldots,{ }_{k}{ }_{k},{ }^{d}\right)$ due to the stochastic uncertainty whose source is the random-number input $\mathbf{u}^{r}$ for that run. Under the assumptions of

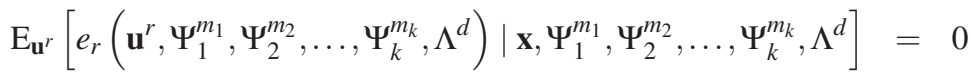

and

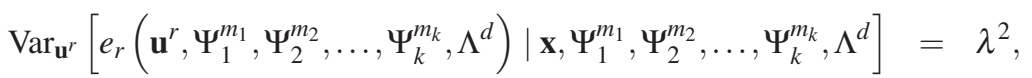

it holds that

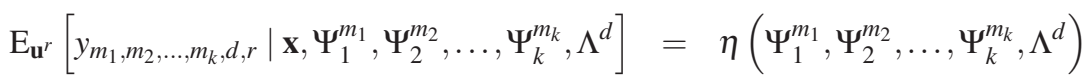

and

$$
\operatorname{Var}_{\mathbf{u}^{r}}\left[y_{m_{1}, m_{2}, \ldots, m_{k}, d, r} \mid \mathbf{x}, \quad \begin{array}{lll}
m_{1} & m_{2}, \ldots, & m_{k} \\
2
\end{array}, d\right]=2
$$

for $m_{i}=1,2, \ldots, M_{i}, i=1,2, \ldots, k, d=1,2, \ldots, D$, and $r=1, \ldots, R$, where 2 represents the stochastic uncertainty. This response surface model is known as the classical random effects model in the statistics literature (Rao 1997). Although its use allows us to estimate stochastic uncertainty ${ }^{2}$, one shortcoming of this response-surface representation is that it assumes constant error variance; i.e., 2 does not depend on the parameters of the multivariate input distribution. The relaxation of this assumption is the subject of our ongoing research.

Next, we assume that

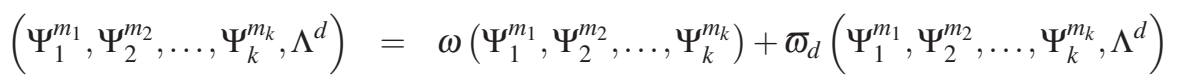

for $m_{i}=1,2, \ldots, M_{i}, i=1,2, \ldots, k$, and $d=1,2, \ldots, D$, where $\quad{ }_{d}\left(\begin{array}{c}m_{1} \\ 1\end{array}, \quad m_{2}, \ldots, \quad{ }_{k}^{m_{k}}, d\right)$ is the deviation of

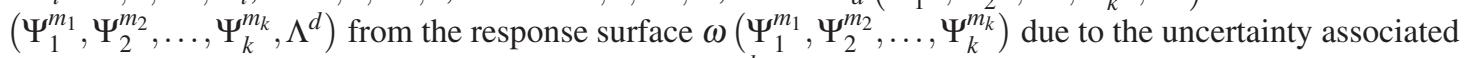
with the underlying dependence structure captured in $d$. Under the assumptions of

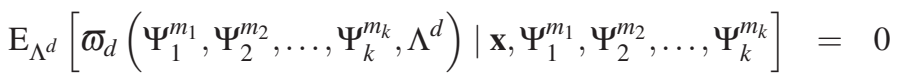

and

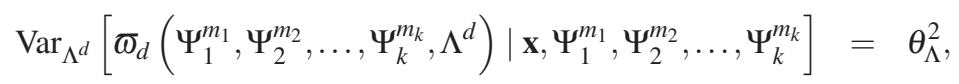

it holds that

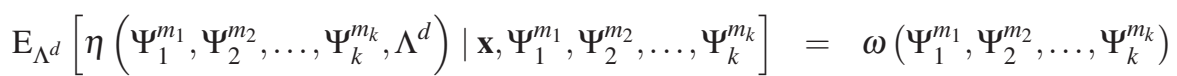

and

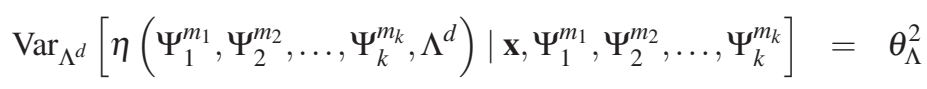

for $m_{i}=1,2, \ldots, M_{i}, i=1,2, \ldots, k$, and $d=1,2, \ldots, D$, where ${ }^{2}$ represents the uncertainty in the dependence structure of the multivariate input process.

Finally, we express the output variability due to the uncertainty associated with the marginal distribution of the $i^{\text {th }}$ simulation input using

$$
i\left(\begin{array}{ccc}
m_{1} \\
1
\end{array}, \begin{array}{l}
m_{2} \\
2
\end{array}, \ldots, \begin{array}{l}
m_{i} \\
i
\end{array}\right)=i-1\left(\begin{array}{ccc}
m_{1} \\
1
\end{array}, \begin{array}{l}
m_{2} \\
2
\end{array}, \ldots, \begin{array}{l}
m_{i-1} \\
i-1
\end{array}\right)+\stackrel{i}{m_{i}}\left(\begin{array}{c}
m_{1} \\
1
\end{array}, \begin{array}{l}
m_{2} \\
2
\end{array}, \ldots, \begin{array}{l}
m_{i} \\
i
\end{array}\right)
$$


for $m_{i}=1,2, \ldots, M_{i}$. Specifically, $\quad{ }_{m_{i}}^{i}\left(\begin{array}{ccc}m_{1} \\ 1\end{array}, \begin{array}{l}m_{2} \\ m_{i}\end{array}, \ldots, \quad \begin{array}{l}m_{i} \\ i\end{array}\right)$ is the deviation of ${ }^{i}\left(\begin{array}{ccc}m_{1} & m_{2} \\ 1 & 2\end{array}, \ldots, \begin{array}{l}m_{i} \\ i\end{array}\right)$ from the response surface ${ }^{i-1}\left(\begin{array}{ccc}m_{1} & m_{2} \\ 1\end{array}, \ldots, \quad \begin{array}{l}m_{i-1} \\ i-1\end{array}\right)$ due to the uncertainty in the $i^{\text {th }}$ marginal distribution parameters. Under the assumptions of

$$
\mathrm{E}_{i}\left[\begin{array}{lll}
i \\
m_{i}
\end{array}\left(\begin{array}{ccc}
m_{1} \\
1
\end{array}, \begin{array}{l}
m_{2} \\
2
\end{array}, \ldots, \begin{array}{l}
m_{i} \\
i
\end{array}\right) \mid \mathbf{x}, \begin{array}{l}
m_{1} \\
1
\end{array}, \begin{array}{l}
m_{2} \\
2
\end{array}, \ldots, \begin{array}{l}
m_{i-1} \\
i-1
\end{array}\right]=0
$$

and

$$
\operatorname{Var}{ }_{i}\left[\begin{array}{ccc}
i \\
m_{i}
\end{array}\left(\begin{array}{ccc}
m_{1} \\
1
\end{array}, \begin{array}{l}
m_{2} \\
2
\end{array}, \ldots, \begin{array}{l}
m_{i} \\
i
\end{array}\right) \mid \mathbf{x}, \begin{array}{l}
m_{1} \\
1
\end{array}, \begin{array}{l}
m_{2} \\
2
\end{array}, \ldots, \begin{array}{l}
m_{i-1} \\
i-1
\end{array}\right]=2_{i},
$$

where $=\mathrm{E}_{1}\left[{ }^{1}\left(\begin{array}{l}1 \\ \end{array}\right) \mid \mathbf{x}\right]$ is an unbiased estimator of the mean posterior response $E_{Y \mid \mathbf{x}}(Y \mid \mathbf{x})$, it holds that

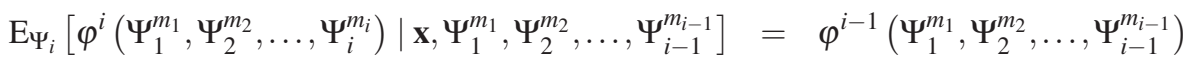

and

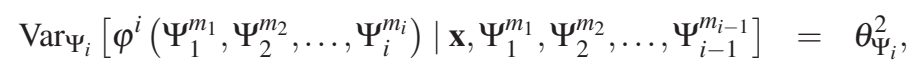

where ${ }^{2}{ }_{i}$ stands for the uncertainty associated with the marginal distribution parameters of the $i^{\text {th }}$ input.

Based on the assumptions (3)-(11), the posterior response variance can be written as $\operatorname{Var}(y \mid \mathbf{x})={ }^{2}+{ }^{2}+$ ${ }_{i=1}^{k} 2_{i}$; i.e., the sum of the three variance components that quantify, respectively, the stochastic uncertainty, the uncertainty in the parameters of the dependence structure, and the uncertainty in the marginal distribution parameters.

Using the simulation output data obtained from the BSRA of Figure 1 and the well-established theory on the classical random-effects model (Rao 1997), we estimate $,{ }^{2},{ }^{2}$, and ${ }^{2}, i=1,2, \ldots, k$ as follows:

$$
\begin{aligned}
& =\bar{y}
\end{aligned}
$$

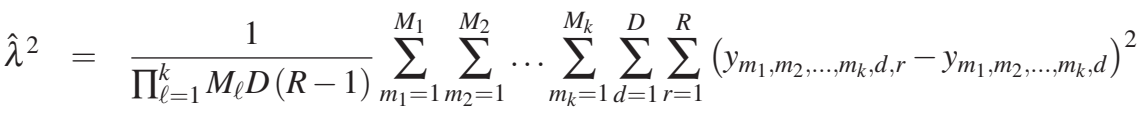

$$
\begin{aligned}
& \wedge^{2}=\frac{1}{D-1}_{d=1}^{D}\left(y_{m_{1}, m_{2}, \ldots, m_{k}, d}-y_{m_{1}, m_{2}, \ldots, m_{k}}\right)^{2}-\frac{\wedge^{2}}{R} \\
& \wedge_{k}^{2}=\frac{1}{M_{k}}{ }_{m_{k}=1}^{M_{k}}\left(y_{m_{1}, m_{2}, \ldots, m_{k}}-y_{m_{1}, m_{2}, \ldots, m_{k-1}}\right)^{2}-\frac{\wedge^{2}}{D}-\frac{\wedge^{2}}{D R} \\
& \wedge_{i}^{2}=\frac{1}{M_{i}-1}{ }_{m_{i}=1}^{M_{i}}\left(y_{m_{1}, m_{2}, \ldots, m_{i}}-y_{m_{1}, m_{2}, \ldots, m_{i-1}}\right)^{2} \\
& -\underbrace{}_{\ell=i+1} \frac{\wedge^{2}}{k_{s=i+1}^{k} M_{s}}-\frac{\wedge^{2}}{k_{s=i+1} M_{s} D}-\frac{\wedge^{2}}{k_{s=i+1} M_{s} D R}, i=k-1, k-2, \ldots, 1
\end{aligned}
$$

Following Zouaoui and Wilson (2003), we construct the $100(1-\quad) \%$ confidence interval for as $\left[y_{\left(\left\lceil M_{1} / 2\right\rceil\right)}\right.$,

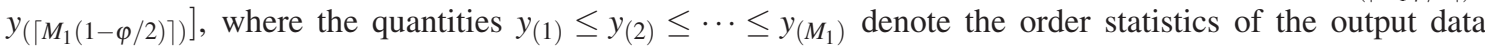
$\left\{y_{m_{1}} ; m_{1}=1,2, \ldots, M_{1}\right\}$ defined in Figure 1 .

\section{EXTENSIONS}

In Section 5.1, we describe how the Bayesian model of Section 2 can be generalized to work for arbitrary marginal distributions, while we discuss the generalization of the model to dependence structures with positive tail dependencies in Section 5.2. 


\subsection{Arbitrary Marginals}

In this paper, we represent the correlated inputs with a multivariate normal distribution. Despite being widely used, multivariate normal distribution is restrictive as it forces the simulation analyst to assume a normal density function for each simulation input. Although we can choose different means and variances for each marginal normal density function, the normal distribution sets the coefficient of skewness to zero and the coefficient of kurtosis to three; thus, it cannot represent inputs with asymmetric probability density functions. However, we can easily extend the presentation in this paper to arbitrary marginal distributions by representing the simulation inputs with the NORTA distribution introduced by Cario and Nelson (1997) for random-vector modeling. The idea is to transform a standard normal base random vector $\mathbf{Z}=\left(Z_{1}, Z_{2}, \ldots, Z_{k}\right)^{\prime}$ into the input random vector $\mathbf{X}=\left(X_{1}, X_{2}, \ldots, X_{k}\right)^{\prime}$ with the transformation $X_{i}=F_{i}^{-1}\left[\left(Z_{i}\right) ; i\right]$ for $i=1,2, \ldots, k$. In this characterization, $F_{i}$ is the marginal cumulative distribution function of input $i$ with parameter vector $i$ and is the cumulative distribution function of the standard normal random variable. The resulting random vector $\mathbf{X}=\left(X_{1}, X_{2}, \ldots, X_{k}\right)^{\prime}$ is said to have a NORTA distribution. This approach works for any marginal distribution; i.e., we may choose $F_{i}$ from any continuous and discrete distribution functions reviewed in Johnson et al. (1994, 2004) and Johnson et al. (2005).

A highly flexible distribution that can be used for modeling the components of the NORTA distribution is the Johnson translation system (Johnson 1949). Recently, Biller and Gunes (2010a) have developed a Bayesian model for estimating the parameters of Johnson's $S_{B}$ and $S_{L}$ distributions. Specifically, they derive Jeffreys' noninformative prior density function for each of the $S_{B}$ and $S_{L}$ distributions, and obtain the posterior density functions of the distribution parameters as a multiplication of the prior density functions and the likelihood functions. The resulting posterior density functions do not belong to well-known distribution functions; therefore, Biller and Gunes (2010a) resort to Markov Chain Monte Carlo techniques for sampling Johnson parameters from their posterior density functions. These sampling algorithms can be employed in the first $k$ steps of the Bayesian simulation replication algorithm of Figure 1 to sample the Johnson parameters from their marginal posterior density functions.

\subsection{Dependence Structures with Positive Tail Dependencies}

The widely used product-moment correlation $\quad(i, j)$ between random variables $X_{i}$ and $X_{j}$ is a measure of linear dependence. Another measure of dependence that has been of interest in recent years is tail dependence; i.e., the dependence in the tails of the joint distributions (Joe 1997). However, many multivariate input models, including the multivariate normal distribution and the NORTA distribution, cannot represent dependence structures with positive tail dependencies because of using a normal copula as the dependence function. A solution for the problem of capturing dependence structures with positive tail dependencies is to replace the (unconditional and conditional) two-dimensional normal copulas of the $C$-vine specification with twodimensional copulas with the ability to represent any pair of lower-tail and upper-tail dependencies. We refer the reader to Joe (1997) and Nelsen (1999) for the class of two-dimensional Archimedean copulas that can be used for this purpose. The development of a Bayesian model that samples dependence parameters from such copulas is the subject of ongoing work. The resulting sampling algorithm can be used in the $(k+1)^{\text {th }}$ step of the BSRA of Figure 1 to sample the tail dependence parameters from their posterior density functions.

\section{CONCLUSION}

In this paper, we consider a stochastic simulation with correlated inputs that are represented by a multivariate normal distribution. We use the Bayesian model of Biller and Gunes (2010) for capturing the uncertainty around the parameters of the multivariate normal distribution (i.e., parameter uncertainty) in this simulation. We then incorporate the Bayesian model into the Bayesian simulation replication algorithm of Zouaoui and Wilson (2003) and decompose the variance of the simulation output into distinct terms representing stochastic uncertainty and parameter uncertainty.

We perform this study under the constant error variance assumption. Ng and Chick (2006) propose a different framework for decomposing the variance of the simulation output into terms related to stochastic 
uncertainty and parameter uncertainty. Their approach is an extension of the frequentist approach of Cheng and Holland (1997) to the Bayesian world; it is based on the asymptotic normality of the confidence intervals of the simulation output. Although their framework assumes that the variance due to stochastic uncertainty does not depend on the system parameters, authors note that the extension of the analysis to the case where the stochastic uncertainty is dependent on the system parameters can be achieved by using the maximum a posteriori estimator of the variance. We are currently investigating how to use the framework of $\mathrm{Ng}$ and Chick (2006) for reducing demand parameter uncertainty in multi-product inventory simulations with correlated demands.

\section{REFERENCES}

Barnard, J., R. McCulloch, and X. Meng. 2000. Modeling covariance matrices in terms of standard deviations and correlations with applications to shrinkage. Statistica Sinica, 10, $1281-1311$.

Barton, R. R. and L. W. Schruben. 2001. Resampling methods for input modeling. In Proceedings of the 2001 Winter Simulation Conference, ed. B. A. Peters, J. S. Smith, D. J. Medeiros, and M. W. Rohrer, 372 - 378. Piscataway, New Jersey: Institute of Electrical and Electronics Engineers.

Bernardo, J. M. and A. F. M. Smith. 1994. Bayesian Theory. New York: John Wiley and Sons, Inc.

Biller, B. and C. Gunes. 2010. Accounting for parameter uncertainty in large-scale stochastic simulations with correlated inputs. Accepted for publication in Operations Research.

Biller, B. and C. Gunes. 2010a. Comparison of least-squares and Bayesian estimation methods for the $S_{L}$ and $\mathrm{S}_{B}$ families of the Johnson translation system. Working Paper, Tepper School of Business, Carnegie Mellon University, Pittsburgh, PA.

Cario, M. C. and B. L. Nelson. 1997. Modeling and generating random vectors with arbitrary marginal distributions and correlation matrix. Working Paper, Department of Industrial Engineering and Management Sciences, Northwestern University, Evanston, IL.

Cheng, R. C. H. and W. Holland. 1997. Sensitivity of computer simulation experiments to errors in input data. Journal of Statistical Computation and Simulation, 57, 327 - 335.

Chick, S. E. 1999. Steps to implement Bayesian input distribution selection. In Proceedings of the 1999 Winter Simulation Conference, ed. P. A. Farrington, H. B. Nembhard, D. T. Sturrock, and G. W. Evans, 317 - 324. Piscataway, New Jersey: Institute of Electrical and Electronics Engineers.

Chick, S. E. 2001. Input distribution selection for simulation experiments: Accounting for input uncertainty. Operations Research, 49, $744-758$.

Cooke, R. M. 1994. Uncertainty in dispersion and deposition accident consequence modeling assessed with performance-based expert judgement. Reliability Engineering and System Safety, 45, 35 - 46.

Hayes, R. H. 1969. Statistical estimation problems in inventory control. Management Science, 15, 686 - 701.

Helton, J. C. 1997. Uncertainty and sensitivity analysis in the presence of stochastic and subjective uncertainty. Journal of Statistical Computation and Simulation, 57, 3 - 76.

Joe, H. 1997. Multivariate Models and Dependence Concepts. London: Chapman and Hall.

Johnson, N. L. 1949. Systems of frequency curves generated by methods of translation. Biometrika, 36, 149 $-176$.

Johnson, N. L., S. Kotz, and N. Balakrishnan. 1994. Continuous Univariate Distributions, Volume 1, 2nd edn. Wiley-Interscience: New York.

Johnson, N. L., S. Kotz, and N. Balakrishnan. 2004. Continuous Univariate Distributions, Volume 2, 2nd edn. Wiley-Interscience: New York.

Johnson, N. L., A. W. Kemp, and S. Kotz. 2005. Univariate Discrete Distributions, 3rd edn. Wiley-Interscience: New York.

Kurowicka, D. and R. Cooke. 2006. Uncertainty Analysis with High Dimensional Dependence Modeling. Wiley Series in Probability and Statistics. John Wiley and Sons, New York.

Law, A. M. 2007. Simulation Modeling and Analysis, 4th edn. New York: McGraw-Hill.

Nelsen, R. B. 1999. An Introduction to Copulas. New York: Springer-Verlag.

Ng, S. H. and S. E. Chick. 2006. Reducing parameter uncertainty for stochastic systems. ACM Transactions on Modeling and Computer Simulation, 16, 26 - 51. 
Raftery, A. E., D. Madigan, and C. T. Volinsky. 1996. Accounting for model uncertainty in survival analysis improves predictive performance (with discussion). In Bayesian Statistics, 5, eds, J. M. Bernardo, J. O. Berger, A. P. David, and A. F. M. Smith. Oxford Press, 323 - 349.

Rao, P. S. R. S. 1997. Variance Components Estimation: Mixed Models, Methodologies, and Applications. Chapman and Hall, London, UK.

Sklar, A. 1959. Fonctions de repartition a $n$ dimensions et leurs margers. Publications de l'Institut Statistique de l"Universitede Paris, 8, 229 - 231.

Zouaoui, F. and J. R. Wilson. 2003. Accounting for parameter uncertainty in simulation input modeling. IIE Transactions, 35, $781-792$.

Zouaoui, F. and J. R. Wilson. 2004. Accounting for input-model and input-parameter uncertainties in simulation. IIE Transactions, 36, 1135 - 1151.

\section{AUTHOR BIOGRAPHIES}

BAHAR BILLER is an assistant professor of Operations Management and Manufacturing at Carnegie Mellon University. Her primary research interest lies in the area of computer simulation experiments for stochastic systems with particular emphasis on multivariate input modeling.

CANAN GUNES is a PhD candidate in the Tepper School of Business at Carnegie Mellon University. Her research interests include the design of large-scale simulations with applications to inventory management, and the applications of Operations Research techniques (e.g., vehicle routing, inventory control) tailored to the problems of non-profit sectors. 